\title{
Behavioural response to exercise in children with attention-deficit/hyperactivity disorder
}

\author{
A J McKune (MMedSci) \\ J Pautz (BTech) \\ J Lombard (BTech)
}

Department of Sport and Physical Rehabilitation Sciences, Technikon Pretoria

\begin{abstract}
Objective. To determine the effect of a moderate intensity exercise programme on the behaviour of children with attention-deficithyperactivity disorder (ADHD).

Design. Thirteen ADHD children $(5-13$ years old, 10 boys and 3 girls) participated in a 5 -week exercise programme $(5$ days a week, 60 minutes of exercise, $20 \mathrm{~min}$ utes at $50-75 \%$ of maximal heart rate). Six ADHD children ( 5 - 13 years old, 3 boys and 3 girls) acted as non-exercising controls. A modified Conner's Parent Rating Scale was used to rate the children's behaviour 1 week before, after 3 weeks of exercise, and immediately after the 5-week period.
\end{abstract}

Main outcome measures. The scale results (total behav lour, attentive behaviour, task orientation, emotional behaviour, motor skills, and oppositional behaviour) were statistically analysed using analysis of variance (ANOVA) with repeated measures. Level of significance was set at $p<0.05$.

Results. There were significant improvements in the behaviour of both the exercise and control groups. Immediately after cessation of the programme the following behaviour categories were significantly improved compared with 1 week before initiation of the programme: total behaviour $(\rho=0.001$, attention $(\rho=0.008)$, emotional $(p=0.01)$, and motor skils $(p=0.004)$.

Conclusion. Contrary to expectations the behaviour of all the ADHD children in the study improved over the 5-week exercise programme. This suggests that if may have been the extra attention paid by parents, guardians

\section{CORRESPONDENCE:}

\section{A J McKune}

Private Bag $X 680$

Pretoria

0001

Tel: $012-3184442$

Fax: 012-318 5801

E-mail: mckunea@techpta.ac.za and exercise leaders or interaction/co-operation. with peers, which altered behavlour rather than the influence of the exercise programme.

\section{Introduction}

Participation in regular low/moderate intensity exercise is associated with positive psychological benefits, such as improvement in mood and the alleviation of anxiety and depression in various populations. ${ }^{4,85.25 .33 .35 .38}$ However, few studies have examined the influence of exercise on behaviour generally, and specifically in individuals with diagnosed behavioural disabilities, such as attention-deficit/hyperactivity disor$\operatorname{der}(A D H D)$.

ADHD is the most common childhood behavioural disorder and is characterised by one or a combination of three behaviours, namely inattention, impulsivity and hyperactivity. ${ }^{1,2.5}$ More than half the children with ADHD have a high rate of disciplinary problems and as many as one-third will progress early into substance experimentation and abuse. ${ }^{7}$ The most commonly prescribed treatment method is the use of psychosomatic medication, such as methylphenidate (Ritalin) ${ }^{14}$ Over $90 \%$ of the child ADHD population in the USA use this medication. However about $20 \%$ experience side-effects including insomnia, decreased appetite, weight loss, headache, heart rate elevation at rest, minor increases in systolic blood pressure, increased crying or mood disturbances. ${ }^{14,27.39}$ These adverse side-effects have led to a need to identify alternative, potentially beneficial treatment options.

Anecdotal reports suggest that exercise is beneficial for children with ADHD. ${ }^{39}$ The few studies that have been published in this area have either been case reports of only one or two children, ${ }^{10,30,36}$ or have focused on whether acute bouts of exercise influences hypothalamic-pituitary-adrenal axis function $^{42}$ or acts as a dopaminergic adjuvant. ${ }^{39}$ Other studies have failed to test a clinically diagnosed population, ${ }^{24,30}$ implement valid behavioural measures, ${ }^{24,30,36}$ and quantify or standardise the exercise stimulus. ${ }^{10,24,36}$ Specifically, minimal research has been conducted on the effect of moderate-intensity, chronic exercise on the behaviour of children with ADHD. Therefore, the primary purpose of this study was to monitor alterations in ADHD behaviour during and following a 5-week moderate intensity exercise programme in children with ADHD. 


\section{Methods}

\section{Subjects}

Thirteen boys and 6 girls clinically diagnosed according to the Diagnostic and Statistical Manual (DSM) III-R guidelines for $A D H D^{2}$ and currently taking methylphenidate $(10-30 \mathrm{mg}$, 1 - 2 times per day) were recruited from a remedial education primary school. This school does not provide formal physical education classes or after school sports and none of the children were participating in regular physical activity programmes outside of the school. The children were all Caucasian and from local middle-income suburbs. They were divided into an experimental, exercise group $(N=13,10$ boys and 3 girls) and a control, non-exercise group ( $N=6,3$ boys and 3 girls). The groups were not randomly allocated due to logistical reasons. The children in the control group were these who were in lift clubs and could not stay after school to exercise.

\section{Experimental design}

\section{Information and training session}

Two weeks prior to commencement of the exercise programme the children and their parents/guardians attended an information session. Each parent/guardian signed an informed consent, previously approved by the institution's ethics committee. They were taught how to complete a Conner's rating scale, as well as to measure the resting heart rate of their children. Parents of both groups were provided with a physical activity data sheet on which they were instructed to keep a record of their children's physical activity every 30 minutes, for 9 hours, after school for the duration of the study. ${ }^{41}$ The data sheet consisted of a table with 18 rows and 4 columns, with the first column indicating the time in half-hour intervals, the second, space for a brief description of the activity, and the third and fourth were for indicating sedentary and active activities respectively. Parents were instructed to place a value of one, every half an hour in the sedentary or active column, depending on whether the activity was sedentary (i.e., TV watching, video games, computer use, talking on phone, homework), or active (i.e. intense play, running, fast walking, swimming or playing in the pool, skating, biking). The sum of each sedentary and active column would then be calculated. The maximum possible score for a child who was sedentary or active for all 9 hours after school could therefore be 18. Based on the total score in the sedentary column, activity levels could be rated as acceptable (score 0 - 3), average (score $4-6$ ), moderately sedentary (score $7-9$ ) and extremely sedentary (score $10-18$ ). Participants had their height measured and were weighed using a balance scale. The sum of triceps brachii and calf skin-folds, measured with a Harpenden calliper, was used to estimate per cent body fat. ${ }^{21}$

\section{Exercise protocol}

\section{Exercise intensity}

For the week prior to commencement of the exercise programme, the exercise group parents recorded their children's supine resting heart rate every morning. The average resting heart rates were used to calculate the individual heart rate zones using the Karvonen equation. ${ }^{23}$ Individual heart rate zones $(50-75 \%$ of maximal heart rate) were programmed into Polar Accurex Plus heart rate monitors. Each child wore a monitor during the exercise sessions, and an alarm was used to alert the exercise leaders when the child's heart rate was either above or below his/her target zone.

\section{Exercise duration}

The children exercised in the afternoon after school from $14 \mathrm{ho0}$ to $15 \mathrm{~h} 00$, five times a week (Monday to Friday) for 5 weeks. Each session was divided into a 15-minute preparation and warm-up phase, a 30-minute exercise phase and 10 minutes of cool down. The cool down phase included Jacobson's progressive relaxation exercises. The aim of the relaxation exercises was to reduce the children's' heart rates back to or close to resting levels. This was so that they were calm and relaxed before going home to do homework or daily chores.

\section{Mode of exercise}

The exercise sessions varied. They included activities such as relay runs, simple plyometric exercises (one-leg hops, tuck jumps), running through a mini obstacle course, a distance run of $1-2$ kilometres, and skipping. The aim was to have the subjects stay within their target hear rate zone for at least 20 minutes in each session.

\section{Behaviour rating scale}

The behaviour rating scale is a modified version of Conners' Parent Rating Scale. ${ }^{6}$ The scale has been validated for screening and assessing behaviour and psychosocial problems, ${ }^{18}$ outcome in children with disruptive behaviour problems," and for measuring behaviours directly related to ADHD. ${ }^{6}$ The scale provides a reliable, accurate, and relatively brief measure of parental perception of children's disruptive behaviour and provides a useful measure for monitoring children's behaviour over time.$^{6}$ It consists of 25 behaviour-related questions, which can be sub-divided into five categories namely attention, motor skills, task-orientation, emotional and oppositional behaviour. Each subject's parents/guardian rated each question as 'not true at all', 'just a little true', 'pretty much true' or 'very much true' by marking the appropriate block with a cross. Questions rated as 'not true at all' scored 4 points, those rated as 'just a little true' scored 3, 'pretty much true' ratings scored 2 points and 'very much true' scored 1. A total score out of 100 was calculated, the higher the score, the better behaved the child. Furthermore, this score was divided into an individual score (out of 20) for each of the five categories. The parents were unaware of the scoring method.

Parents of children in the experimental and control group were asked to complete the behaviour rating scale 1 week before commencement of the exercise programme, after 3 weeks and immediately after cessation of the exercise programme at 5 weeks.

\section{Statistics}

To determine any significant changes in behaviour, all variables were subjected to an analysis of variance (ANOVA) with repeated measures. Tukey's post-hoc tests were used to determine significant differences attributed to time. Data were analysed using commercial software (SAS Institute, Cary, 
NC). The level of significance was set at $p<0.05$.

\section{Results}

The children's physical characteristics were (mean \pm SD): age, experimental group (10.8 \pm 1.9 years), control group (11.2 \pm 1.5 years); weight, experimental group $(40.42 \pm 3.88 \mathrm{~kg})$, control group (38.11 $\pm 5.11 \mathrm{~kg})$; height, experimental group $(144.65 \pm 4.62 \mathrm{~cm})$, control group $(146.38 \pm 8.15 \mathrm{~cm})$; body fat, experimental group (19.65 $\pm 4.33 \%)$, control group (22.43 $\pm 6.35 \%)$. The physical activity records of the control group indicated that there was no increase in their daily levels of physical activity during the duration of the study. The average activity scores for the control children ranged between 10 and 18 (extremely sedentary) for the duration of the study.

\section{Behaviour}

There were no significant differences between the experimental and control groups with regard to behavioural ratings over the 5 weeks. However, there were significant alterations in the behaviour of both groups when the results were combined.

Immediately after cessation of the programme the following behaviour categories were significantly improved compared with 1 week before initiation of the exercise programme: total behaviour $(p=0.001)$, attention $(p=0.008)$, emotional $(p=0.01)$, and motor skills $(p=0.004)$ (Fig. 1). Task orientation and oppositional behaviour (Fig. 2) were not significantly altered after the 5 weeks. However, the trend was similar to that which occurred in the other categories where behaviour improved over time.

\section{Discussion}

Although limited research has been performed on the role of exercise in improving the behaviour of children with ADHD, researchers have noted the positive role that increased exercise levels play in the treatment of anxiety ${ }^{32,34,35,38}$ depression, stress $^{32-34}$ and enhancing general mood state. ${ }^{34,35,38}$ Based on this previous research on mood and exercise, and anecdotal reports from parents and teachers, it was hypothesised that an exercise programme would significantly improve the behaviour of children with ADHD compared with that of non-exercising controls. The present study therefore, examined the alterations in the behaviour of children with ADHD during and after a 5-week moderate-intensity exercise programme. Interestingly, no significant differences were found between the behaviour of the exercising children and those of the control group over the 5 weeks. However, there were significant improvements in the behaviour of both groups in four out of six behaviour categories. With regard to the exercise group it is thought that exercise-induced changes in neurotransmitters may be a possible explanation for the improved behaviour.

Dysfunctional brain neurotransmitter pathways have been shown to contribute to the behavioural and neuropsychological features of ADHD. ${ }^{20,26}$ Exercise has been shown to alter neurotransmitter levels in the blood. ${ }^{37,42}$ it has therefore been hypothesised that exercise may also facilitate the efficient use of neurotransmitters in the brain neuronal circuits, thereby improving neurophysiological functioning and the behaviour of
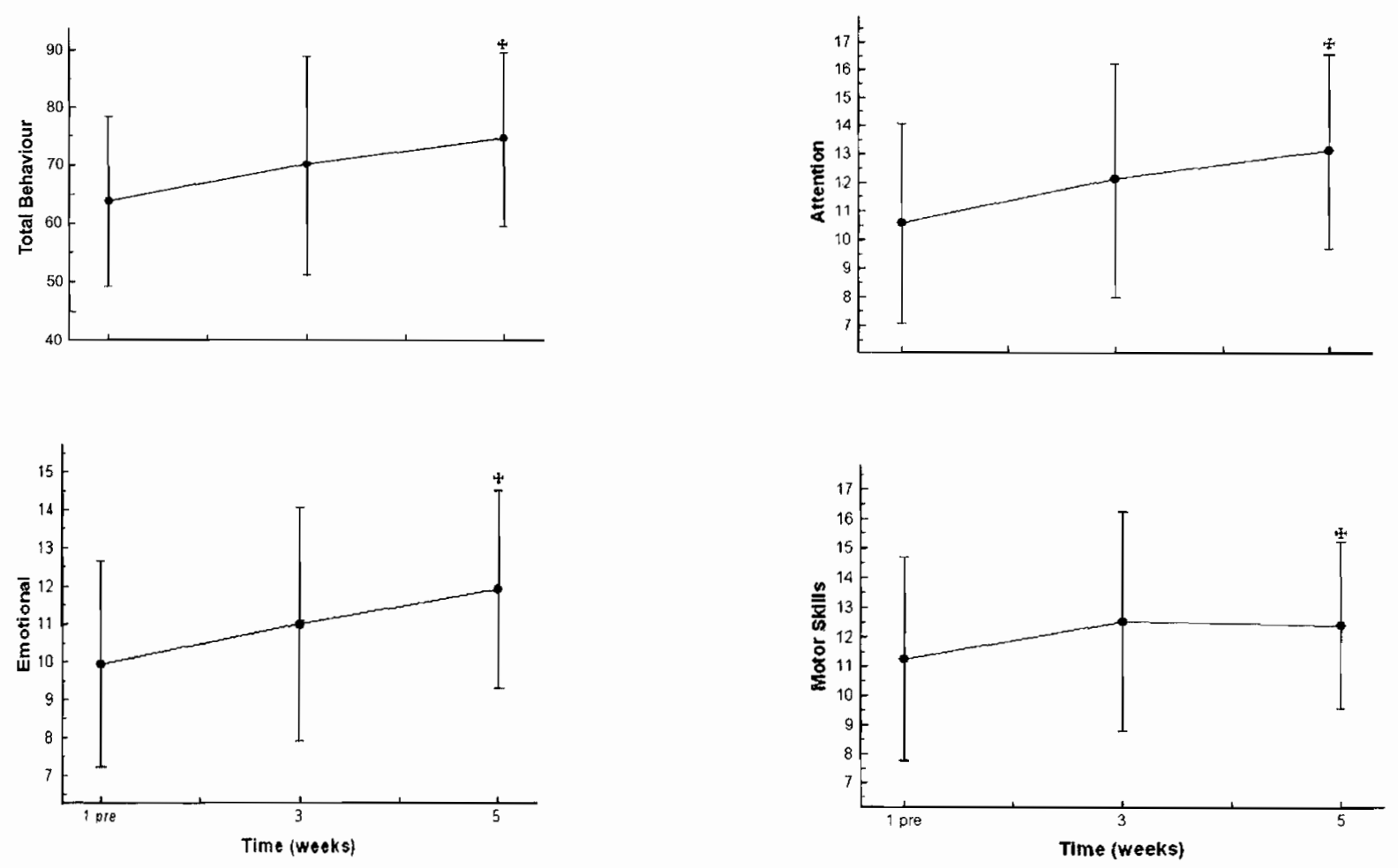

Fig. 1. Alterations in total behaviour, attentive behaviour, emotional behaviour and motor skills (mean $\pm S E M,+=p<0.05)$ for 19 subjects during and immediately after 5 weeks of participation in the study. 

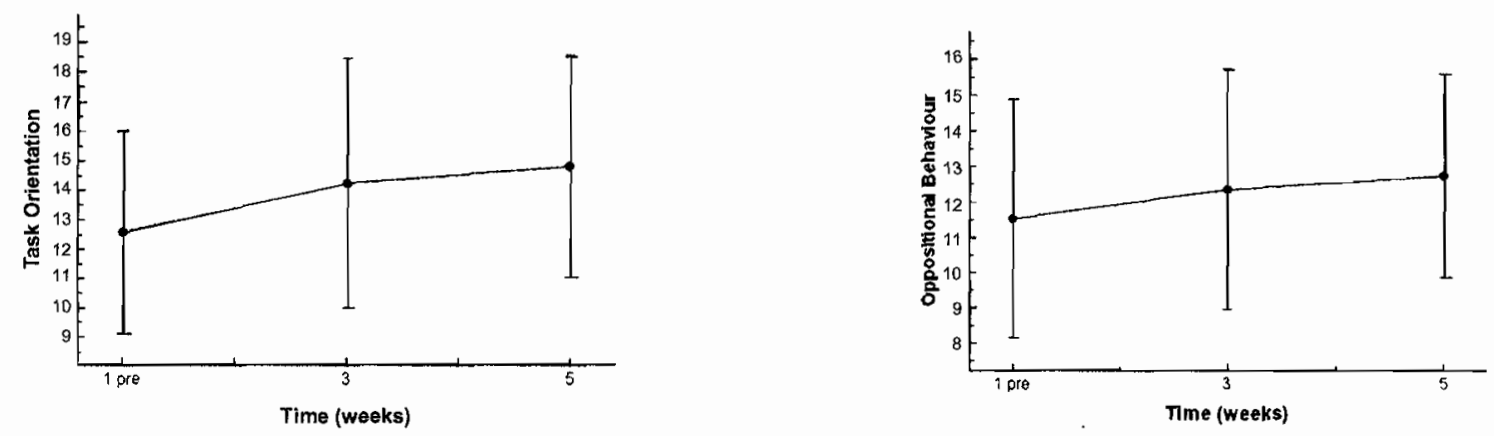

Fig. 2. Alterations in task orientation and oppositional behaviour (mean \pm SEM) for 19 subjects during and immediately after 5 weeks of participation in the study.

children with ADHD..$^{41}$ The major limitation of this hypothesis is firstly that an alteration in the level of neurotransmitters or precursors in the blood does not necessarily reflect the changes that are occurring in the brain, and secondly that research examining the effect of exercise on brain neurotransmitter release is essentially limited to animal models or postmortem methods. ${ }^{28}$ Despite these limitations further support for the hypothesis comes from research using brain microdialysis to measure the effect of exercise on brain neurotransmitter levels in vivo. ${ }^{28}$ Although this research is based on a rat model, there is evidence that 20 minutes of exercise on a treadmill significantly increases dopamine release in rat striatum. ${ }^{15.16}$ Similarly, elevated dopamine agonist levels after acute treadmill running ${ }^{12,17}$ and forced swimming ${ }^{43}$ have also been reported, and 6 weeks of training, 5 days/week, increasing training time and speed from 30 minutes, $19 \mathrm{~m} / \mathrm{min}$ to 80 minutes, $26 \mathrm{~m} / \mathrm{min}$ by the final week, increased dopamine and norepinephrine in the striatum of rats. ${ }^{29}$ Evidence from the rat model regarding elevated brain dopamine and norepinephrine levels, although not ideal, suggests that the dose of exercise used in the present study may be sufficient to result in adaptive responses in the neurotransmitter system. It is suggested, however, that future studies should investigate the effect of different doses of exercise on ADHD behaviour.

To date five published studies have reported the effects of physical activity/exercise on children with attentional and/or hyperactivity disorders. ${ }^{10,24,30,36,39}$ In the study by Tantillo et al., ${ }^{39}$ the major weaknesses of the previous studies were addressed. The authors used clinically diagnosed ADHD children, quantified the exercise dose, implemented valid behavioural measures and used measures related to aetiological mechanisms of ADHD that might explain the effect of exercise. The study found that a hallmark feature of ADHD, motor impersistence (inability to sustain motor acts such a maintaining a conjugate gaze, keeping the mouth open, protruding the tongue, fixing the eyes centrally, or holding the eye lids shut) was reduced in boys but not girls after a $\mathrm{VO}_{2 \text { peak }}$ test. Motor impersistence is related to the behavioural disinhibition often seen in children with $A D H D$ and a reduction may result in improved behaviour. ${ }^{40}$

Tantillo et al..$^{39}$ also attempted to measure the effect of the exercise on two indirect measures of brain dopaminergic activity namely spontaneous eye blinks and the acoustic starthe eye blink response (ASER) ${ }^{9,22}$ The results showed that boys had increased spontaneous blink rate and decreased
ASER after a $\mathrm{VO}_{2 \text { peak }}$ test. This would indicate increased exercise-induced brain dopaminergic activity. ${ }^{9.22}$ The study by Tantillo et $a l^{39}$ therefore provides preliminary evidence of the potential efficacy of acute exercise in helping to manage ADHD in boys. Additionally it highlighted an important issue relating to gender differences in ADHD symptomatology ${ }^{13,31}$ that may influence the therapeutic benefits of exercise on ADHD behaviour..$^{39}$ Although further research is required in this area, the possibility therefore exists in the present study that a gender difference in response to the nature and/or dose of exercise may have influenced the results.

The study has limitations, which provide additional explanations, other than exercise-induced neurotransmitter changes, for improved behaviour in ADHD children. Firstly, the small sample size, particularly in the control group, which made it difficult to randomise the children, may have influenced the results. Secondly, the influence of increased attention paid by parents to their children during the rating of their behaviour and activities was not controlled for. Research has shown that the presence of ADHD in children is closely associated with disrupted parent-child relationships ${ }^{21}$ and parentchild conflicts. ${ }^{3}$ Therefore, a possible explanation for the results for both groups may simply be the fact that parents/guardians/exercise leaders spent more time or paid more attention to the children for the duration of the study. Future research in this area should address this concept. A third limitation of the study was possible influence and social interaction within and between the exercise and control groups, as the children attended the same school. Children with $A D H D$ are known to experience difficulty in peer relationships. ${ }^{19}$ A possible explanation for improved behaviour may therefore be that the exercise sessions encouraged co-operation in group situations and fostered tolerance and acceptance for other children. Hoza et al. ${ }^{19}$ have reported that friendship forming in ADHD children is associated with improved behaviour. The social interactions in the exercise group during the exercise sessions, as well as during school with the control group may therefore have resulted in improved behaviour in both groups. Finally the fourth limitation of the study was a lack of placebo intervention for the control group. Future studies should address this issue by providing a placebo intervention at the same time of day as the exercise sessions. An example of placebo sedentary activities may be recreational hobbies activities such as arts and crafts, with peers and a leader present for the same duration as the exercise sessions. 


\section{Conclusion}

The objective of the study was primarily to validate the anecdotal claims that exercise will improve the behaviour of children with $A D H D$. There is limited information available on the effect of exercise on ADHD behaviour generally, and specifically moderate-intensity, chronic exercise. In this study it was found that the behaviour of children with ADHD improved over 5 weeks irrespective of their physical activity level. The result suggests that the increased attention of parents/guardians, exercise leaders, or interaction/co-operation with peers, may be the additional 'treatment' required to improve the behaviour of children with ADHD. Future studies should control for these influences to determine the exact role that exercise may play in altering $A D H D$ behaviour.

\section{REFERENCES}

1. American Psychiatric Association. Diagnostic and Statistical Manual of Mental Disorders. 4th ed. Washington, DC.: American Psychiatric Association, 2000.

2. American Psychiatric Association. Diagnostic, and Statistical Manual of Mental Disorders. 3rd ed. Washington DC:: American Psychiatric Association, 1987.

3. Burt SA, Krueger RF, McGue M, Lacono W. Parent-child conflict and the comorbidity among childhood externalizing disorders. Arch Gen Psychiatry 2003; 60: $505-13$.

4. Byrne A, Byrne DG. The effect of exercise on depression, anxiety and other mood states: A review. J Psychosom Res 1993; 37: 565-74.

5. Cantwell DP. Attention deficit disorder: A review of the past 10 years. $J \mathrm{Am}$ Acad Child Adolesc Psychiatry 1995; 35: 978-87.

6. Conners CK, Sitarenios G, Parker JD, Epstein JN. The revised Conners' Parent Rating Scale (CPRS-R): factor structure, reliability, and criterion validity. J Abnorm Child Psychol 1998; 26: 257-68.

7. Craft $\mathrm{DH}$. Learning disabilities and attentional deficits. In: Winnick JP, ed. Adapted Physical Education and Sport. 3rd ed. Champaign, III.: Human Kinetics, 2000: 127-41.

8. DiLorenzo TM, Bargman EP, Stucky-Ropp R, Brassington GS, Frensch PA, LaFontaine $T$. Long-term effects of aerobic exercise on psychological outcomes. Prev Med 1999; 28: 75-85.

9. Elsworth JD, Lawrence MS, Roth $\mathrm{RH}$, et al. D1 and D2 dopamine receptors independently regulate spontaneous blink rate in the vervet monkey. J Pharmacol Exp Ther 1991; 259: 595-600.

10. Etscheidt MA, Ayllon T. Contingent exercise to decrease hyperactivity. Psychotherapy Journal 1987; 4: 192-8.

11. Fischer M, Newby RF. Assessment of stimulant response in ADHD children using a refined multimethod clinical protocol [special issue on child psychopharmacology]. J Clin Child Psychol 1991; 20: 232-44.

12. Freed $C R$, Yamamoto BK. Regional brain dopamine metabolism: a marker for the speed, direction, and posture of moving animals. Science 1985; 229: $62-5$.

13. Gaub M, Carlson CL. Gender differences in ADHD: a meta-analysis and critical review. J Am Acad Child Adolesc Psychiatry 1997; 36: 1036-45.

14. Greenhill LL. Pharmacologic treatment of attention deficit hyperactivity disorder. Pediatric Psychopharmacology 1992; 15: 1-27.

15. Hattori S, Li Q, Matsui N. Treadmill running combined with microdialysis can evaluate motor deficit and improvement following dopaminergic grafts in 6 OHDA lesioned rats. Neurological Neuroscience 1993; 6: 65-72.

16. Hattori $S$, Naoi $M$, Nishino $H$. Striatal dopamine turnover during treadmill running in the rat: relation to the speed of running. Brain Res Bull 1994; 35: 41-9.

17. Heyes MP, Garnett ES, Coates G. Nigrostriatal dopaminergic activity is increased during exhaustive exercise stress in rats. Life Sci 1988; 42: 153742.

18. Horn WF, lalongo N, Popovich S, Peradotto D. Behavioural parent training and cognitive-behavioural self-control therapy with ADD-H children Comparative and combined effects. J Clin Child Psychol 1987; 16: 57-68.

19. Hoza B, Mrug S, Pelham WE jun., Greiner AR, Gnagy EM. A friendship intervention for children with Attention-Deficit/Hyperactivity Disorder: preliminary findings. Journal of Attention Disorders 2003; 6: 87-98.

20. Hynd GW, Hern KL, Novey ES, et al. Attention deficit-hyperactivity disorder and asymmetry of the caudate nucleus. Journal of Child Neurology 1993; 8: 339-47.

21. Johnston C, Mash EJ. Families of children with attention-deficit/hyperactivity disorder: review and recommendations for future research. Journal of Clinical Child Neurology and Family Psychology Review 2001; 4: 183-207.

22. Karson CN. Spontaneous eye-blink rates and dopaminergic systems. Brain 1983; 106: 643-53.

23. Karvonen $\mathrm{J}$, Vuorimaa $\mathrm{T}$. Heart rate and exercise intensity during sports activities: Practical application. Sports Med 1988; 5: 303-12.

24. Klein SA, Deffenbacher JL. Relaxation and exercise for hyperactive impulsive children. Percept Mot Skills 1977; 45: 1159-62.

25. Lane $A_{1}$ Crone-Grant $D$, Lane $H$. Mood changes following exercise. Percept Mot Skills 2002; 94: 732-4.

26. Lou. HC, Henriksen L, Bruhn P, Borner. H, Nielsen JB. Striatal dysfunction in attention deficit and hyperkinetic disorder. Arch Neurol 1989; 46: 48-52.

27. Masellis M, Basile VS, Muglia P, Ozdemir V, Macciardi FM, Kennedy JL. Psychiatric pharmacogenetics: personalizing psychostimulant therapy in attention-deficit/hyperactivity disorder. Behav Brain Res 2002; 130: 85-90,

28. Meeusen R, Piacentini MF, De Meirleir K. Brain microdialysis in exercise research. Sports Med 2001; 31: 965-83.

29. Meeusen R, Smolders I, Sarre S, et al. Endurance training effects on neurotransmitter release in rat striatum: an in vivo microdialysis study. Acta Physiol Scand 1997; 159: 335-41.

30. Molloy GN. Chemicals, exercise and hyperactivity: a short report International Joumal of Disability, Development and Education 1989; 36: 57 61

31. Morgan AE, Hynd GW, Riccio CA, Hall J. Validity of DSM-IV ADHD predominantly inattentive and combined types: relationship to previous DSM diagnoses/subtype differences. J Am Acad Child Adolesc Psychiatry 1996; 35: 325-33.

32. Norris R, Carroli D, Cochrane R. The effects of physical activity and exercise training on psychological stress and weil-being in an adolescent population. J Psychosom Res 1992; 36: 55-65.

33. Paluska SA, Schwenk TL. Physical activity and mental health: Current concepts. Sports Med 2000; 29: 167-180.

34. Potgieter JR. Sport Psychology: Theory and Practice. Stellenbosch: Institute for Sport and Movement Studies, University of Stellenbosch, 1997.

35. Raglin JS, Morgan WP. Influence of exercise and quiet rest on state anxiety and blood pressure. Med Sci Sports Exerc 1987; 19: 456-83

36. Silverstein JM, Allison DB. The comparative efficacy of antecedent exercise and methylphenidate: a single-case randomized trial. Child Care Health Dev 1994; $20: 47-60$

37. Spencer TJ, Biederman J, Wilens TE, Faraone SV. Novel treatments for attention-deficit/hyperactivity disorder in children. J Clin Psychiatry 2002; 63: $16-22$.

38. Steptoe A, Cox S. Acute effects of aerobic exercise on mood. Health Psychol 1988; 7: 329-40.

39. Tantillo M, Kesich CM, Hynd GW, Dishman RK. The effects of exercise on children with attention-deficit hyperactivity disorder. Med Sci Sports Exerc 2002; 34: 203-12.

40. Voeller KS, Heilman KM. Motor impersistence in children with attention deficit hyperactivity disorder: evidence for right hemisphere disfunction. Ann Neurol 1998; 24: 323.

41. Wendt MS. The Effect of an Activity Program Designed with Intense Physical Exercise on the Behavior of AD/HD Children. Buffalo: State University of New York, 2000.

42. Wigal SB, Nemet D, Swanson JM, et al. Catecholamine response to exercise in children with attention deficit hyperactivity disorder. Pediatr Res 2003; 53: 756-61.

43. Yadid $\mathrm{G}$, Overstreet $\mathrm{DH}$, Zangen A. Limbic dopaminergic adaptation to a stressful stimulus in a rat model of depression. Brain Res 2001; 896: 43-7. 